\title{
Análisis y proyección de los rendimientos accionarios de Pfizer, en el período 2018-2020, mediante redes neuronales diferenciales
}

Alfonso Aja Kindelan Socio fundador de El Pescau

Leovardo Mata Mata Universidad Anáhuac México

Jaime Humberto Beltrán Godoy Universidad Anáhuac México

Artículo recibido el 17 de diciembre de 2018 y aprobado el 24 de abril de 2019

\section{Resumen}

En este trabajo se utiliza una red neuronal diferencial (DNN, por sus siglas en inglés) para proyectar los rendimientos accionarios de Pfizer en el período 2018-2020. El modelo emplea datos trimestrales, al cierre del periodo, del precio de la acción de la empresa (P), ventas netas (VN), activos totales (AT) y cuentas por cobrar (CC). Los resultados señalan una bondad de ajuste superior de las DNN frente a los métodos convencionales, pues el error en el pronóstico out sample es inferior al $5 \%$. Este hallazgo contribuye con evidencia empírica para afirmar que las DNN ofrecen mayor robustez predictiva de los rendimientos accionarios de Pfizer.

Palabras clave: redes neuronales, pronóstico, rendimientos accionarios. Clasificación JEL: C45, C51. 


\section{Abstract}

In this paper, a differential neural network (DNN) is used to project Pfizer's stock returns in the 2018-2020 period. The model uses quarterly data, at the end of the period, the price of the company's stock (P), net sales (NS), total assets (TA) and accounts receivable (AR). The results are compared with the classic regression models and there is evidence of the superior goodness of fit of the DNN, compared to conventional methods, since the error in out sample forecast is less than $5 \%$.

Keywords: neural networks, forecast, stock returns.

JEL Classification: C45, C51. 


\section{Introducción}

Pfizer Inc. es una empresa dedicada al desarrollo, producción y distribución de medicamentos en la industria farmacéutica, con altos ingresos en los últimos años.

Sin embargo, debido a la elección del nuevo presidente Donald Trump en Estados Unidos, existe mucha incertidumbre sobre el futuro de la empresa, ya que él ha propuesto disolver el Obama Care, una reforma implementada por su antecesor (enfocada en disminuir el costo y mejorar el acceso a la salud, mediante regulaciones e impuestos), por lo que el análisis del precio de la acción que se realiza en este trabajo resulta relevante y pertinente.

Por ello, se elaborará un breve análisis financiero de la empresa Pfizer, con lo cual se tendrá un esquema que permita proyectar los rendimientos accionarios de la organización, mediante redes neuronales diferenciales, hacia el período 2016-2020.

El proyecto está dividido en tres apartados, que se describen a continuación.

En el primero, se presentará el marco teórico que respalda el enfoque de estimación utilizado. En el segundo, se describirá brevemente la metodología, siguiendo los esquemas y especificaciones descritos en el primer apartado. Luego, en la tercera sección se analizará el precio de la acción para los años 2016-2020, considerando las variables de tendencia determinística, ventas netas, activos totales, las cuentas por cobrar y un término de perturbación aleatoria, pues se vinculan con el precio de la acción de Pfizer, como sugiere el enfoque de Londoño, Lopera y Restrepo (2010).

Finalmente, las proyecciones financieras del precio de la acción y su volatilidad se calcularán mediante las premisas heurísticas de una red neuronal diferencial (DNN, por sus siglas en inglés) y la información histórica disponible. El objetivo es predecir la posible dirección que la empresa puede tomar en los próximos cinco años, de acuerdo con el comportamiento del precio de su acción. Dicho ejercicio no solo es útil para estudiar los rendimientos accionarios de la empresa, sino también como herramienta para la toma de decisiones de inversión.

\section{Marco teórico}

La teoría de la estructura de capital trata sobre las proporciones de financiamiento, en el costo explícito y el implícito de la deuda y del costo de capital. El análisis correcto de la estructura de capital busca explicar su comportamiento a lo largo del tiempo, donde los flujos de efectivo son un factor clave para estudiar la empresa. Esto es relevante porque se vinculan 
los flujos con el costo promedio ponderado de capital (WACC) y con el modelo CAPM, así que se puede establecer un puente entre diversas variables contables de la empresa y los rendimientos accionarios (Ross et al., 2012).

Asimismo, la teoría de riesgo de mercado busca cuantificar las pérdidas para observar grados de sensibilidad en relación con situaciones de mercado. La teoría de riesgo de mercado tiene como punto central una frontera de eficiencia, que maximiza rendimientos y minimiza riesgo. Aunque estos temas se aplican usualmente a los portafolios de inversión, puede emplearse dicho enfoque para valorar el grado de incertidumbre de un activo, en particular el precio de una acción (Lara, 2009).

A este respecto, si bien es cierto que el precio de una acción se encuentra sujeto al juego de la oferta y la demanda, asumir riesgos y enfrentarse a situaciones inesperadas también desempeñan un papel clave. En ese caso, el precio puede fluctuar de un período a otro, sin que se tengan identificados claramente los factores de riesgo causales (Sirghi, 2012).

Existen básicamente dos componentes: un elemento sistemático y otro no sistemático. El primero puede analizarse mediante la consideración de aquellas variables que, según la teoría de la estructura de capital, influyen y determinan parte del precio de la acción, como son ventas netas, activos totales, cuentas por cobrar, entre otras variables financieras, así como las razones correspondientes (Tricker, 2009).

El segundo componente no es observable y usualmente puede tratarse como una perturbación aleatoria, la cual puede simularse o al menos se busca controlar para que el grado de incertidumbre sea menor (Hornik, Stinchcombe y White, 1989).

La principal ventaja que se reporta en la bibliografía, en relación con las dos teorías señaladas, es que tienen un soporte teórico amplio que permite medir diversos aspectos de la empresa y el mercado. No obstante, su principal debilidad surge de la complejidad de uso de sus elementos, ya que las relaciones matemáticas oscurecen la interpretación de los resultados o encarecen los puentes entre la dinámica real de la empresa y los modelos econométricos con distribuciones de probabilidad poco prácticas (Venegas, 2013).

Los dos enfoques teóricos anteriores son necesarios para justificar el vínculo entre los rendimientos accionarios de Pfizer y las variables internas de la empresa, tales como las ventas netas (VN), los activos totales (AT) y las cuentas por cobrar (CC), sin importar la periodicidad de la información. En particular, este documento realiza las estimaciones con datos trimestrales, al cierre del período, pues la disponibilidad de la información de todas las variables a nivel semanal o diario es limitada.

Ahora bien, es importante señalar que en economía y finanzas se han vinculado estos dos enfoques mediante diversas técnicas numéricas, entre las 
que sobresalen mínimos cuadrados ordinarios, modelos ARIMA-GARCH y redes neuronales artificiales - ANN, por sus siglas en inglés - (León et al., 2018).

Las ANN han presentado un alto desempeño para la predicción de rendimientos de diversos activos financieros en comparación con otras técnicas estadísticas (Misas, López y Borrero, 2002). Esto se debe a que las ANN son técnicas heurísticas que realizan aproximaciones según el comportamiento histórico no lineal de las series de datos (Ospina y Zamprogno, 2003; León et al., 2018).

En particular, las ANN se han empleado en el estudio de los mercados financieros desde mediados de los años ochenta. Por ejemplo, en Estados Unidos comenzaron a utilizarse en 1985 para el análisis y pronóstico del sector bursátil, donde las redes neuronales perceptrón multicapa (ANN-M, por sus siglas en inglés) se desempeñaron mejor que los modelos de regresión lineal, aunque no al nivel esperado por los analistas (White, 1988).

Desde entonces, la aplicación de las ANN se ha popularizado en diferentes áreas, tanto de las ciencias sociales como de las ciencias naturales. Sin embargo, en cada caso los resultados son heterogéneos. La bondad de ajuste depende de la cantidad de información disponible para entrenar a la ANN, además de que la implementación numérica y la supervisión del entrenamiento no es simple (Landassuri et al., 2013).

Diversos autores, tales como Ortiz, Llanos y Herrera (2013) y Pao (2008), han combinado las ANN con diversos modelos para calibrar redes neuronales robustas, tales como medias móviles, suavizamiento exponencial, análisis de regresión, modelos ARIMA-GARCH e incluso con especificaciones más sofisticadas como datos de panel, vectores autorregresivos (VAR) y lógica difusa.

Las ventajas que se obtienen al combinar las ANN con algún procedimiento clásico se pueden clasificar en cuatro: aprendizaje adaptativo, autoorganización, pronósticos robustos y tiempo de computo manejable para la toma de decisiones (Ortiz, 2017).

Además, resulta conveniente mencionar que han aparecido variantes de las ANN, dependiendo del tipo de datos que se va a estudiar, así como de las variables disponibles para construir el modelo heurístico asociado con la red. Entre los diversos tipos de ANN sobresalen las redes diferenciales, porque emplean técnicas de análisis de estabilidad de acuerdo con la función de Liapunov, que es una herramienta de la teoría de sistemas dinámicos que se utiliza para establecer el desarrollo de las leyes de aprendizaje y controlar la estabilidad de la ANN (Ortiz, Llanos y Herrera, 2013).

En este trabajo, se emplea una red neuronal diferencial, ya que se han obtenido resultados robustos para diversos mercados accionarios, tales 
como Nasdaq y el índice S\&P-500 (Moghaddam, Moghaddam y Esfandyari, 2016), así como el Índice de Precios y Cotizaciones (IPC-35; Ortiz, 2017).

En el siguiente apartado se detalla con más cuidado la definición y estructura de una ANN.

\section{Redes neuronales}

En este apartado, se explica la metodología de redes neuronales y se explora el comportamiento de los rendimientos accionarios de Pfizer, en el período 1995-2017. El modelo emplea datos trimestrales, al cierre del período, del precio de la acción de la empresa $(\mathrm{P})$, ventas netas $(\mathrm{VN})$, activos totales (AT) y cuentas por cobrar (CC). Es importante señalar que se estima también una regresión en series de tiempo, con fines comparativos, para establecer las ventajas de pronóstico de las ANN frente a los métodos convencionales.

Las ANN son modelos matemáticos heurísticos que buscan emular el funcionamiento del cerebro humano, pues establecen relaciones no lineales entre las entradas y las salidas de un proceso específico, pero bajo un enfoque de aprendizaje adaptativo, auto-organización y funcionamiento paralelo (Toro, Mejía y Salazar, 2004).

Este tipo de modelos se ha empleado con éxito en el reconocimiento de patrones, planeación, pronóstico y optimización (Villada, Muñoz y García, 2012). La ventaja comparativa de las ANN, frente a los algoritmos convencionales, radica en que no se ejecutan instrucciones secuenciales, sino que se trabaja en paralelo a las entradas y salidas que se presentan (Ortiz, Llanos y Herrera, 2013).

Las ANN no corren un programa, sino que aprenden y procesan información simultáneamente con base en restricciones y ponderaciones que se actualizan constantemente. No obstante, como no se ejecuta un código paso a paso, resulta más difícil detectar errores (Toro, Mejía y Salazar, 2004).

El esquema de redes neuronales más utilizado se llama propagación hacia delante (perceptrón multicapa) y se presenta en la figura 1. Las variables de entrada $X_{1}, X_{2}, X_{3}, X_{4}$ y $X_{5}$ se procesan en las neuronas (unidades de proceso) $F T_{0}, F T_{1}$ y $F T_{2}$ donde se establece un sistema de retroalimentación entre las entradas y las salidas $O_{1}$ y $O_{2}$. Las unidades de proceso son funciones que relacionan todas las variables bajo algún esquema teórico o empírico.

La magnitud de los valores estimados en las unidades de proceso cobra relevancia dentro de la red, mediante diversos umbrales de decisión. Los umbrales de decisión y activación emplean distribuciones de probabilidad, siendo la más utilizada la función sigmoide dada por:

$$
f_{k}\left(F T_{k}\right)=\frac{1}{1+\exp \left(-F T_{k} / \sigma\right)}
$$


donde $\sigma>0$ es un parámetro de ajuste y $k$ el número de capas (etapas de procesamiento) que se tienen en la ANN.

Figura 1. ANN clásica

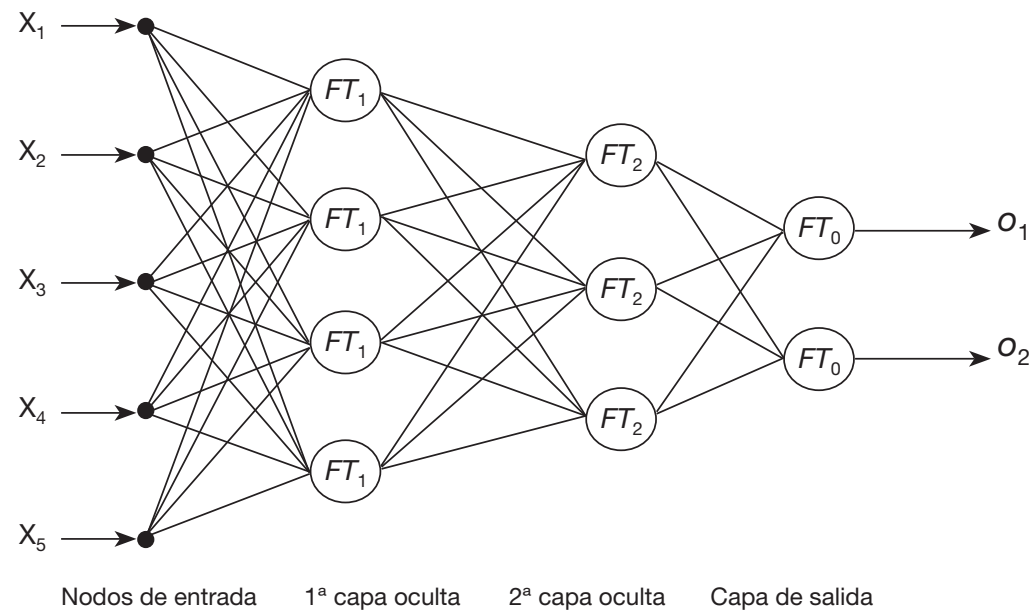

Fuente: Villada, Muñoz y García (2012).

En términos sencillos, puede pensarse que las salidas quedan expresadas como una combinación ponderada de las entradas que han sido analizadas por las neuronas:

$$
O_{i}=\sum_{j} w_{i j} f_{j}\left(F T_{j}\right)
$$

donde $w_{i j}$ son las ponderaciones para $i=1,2, \ldots, n$ salidas y $j=1,2, \ldots, m$ entradas.

El número de variables de entrada depende directamente de la información disponible, mientras que el número de neuronas empleadas es igual al número de clases en que puedan analizarse los conjuntos de datos (Foix y Weber, 2017). Las unidades de una capa se conectan unidireccionalmente con las de la siguiente, en general todas con todas, sometiendo sus salidas a la multiplicación por un peso $w_{i j}$ que es diferente para cada una de las conexiones (Toro, Mejía y Salazar, 2004).

Más aún, cuando se trata de una red neuronal artificial diferencial se asume que tanto los valores de entrada como los de salida pueden ser medibles, por lo que forman parte vital del entrenamiento de la red (Landassuri et al., 2013).

Matemáticamente, la red neuronal diferencial puede describirse mediante una ecuación diferencial (Cabrera et al., 2007) dada por 


$$
\dot{x_{t}}=A x_{t}+W_{1, t} \sigma\left(x_{t}\right)+W_{2, t} \varphi\left(x_{t}\right) u_{t}
$$

Donde $x_{t} \in \mathbb{R}^{n}$ es el estado de la DNN, $u_{\mathrm{t}}$ es la entrada que proviene de los modelos de calibración, $W_{1, t} \in \mathbb{R}^{n \times k}$ es la matriz de pesos de la capa de estados de retroalimentación, $W_{2, t} \in \mathbb{R}^{n \times k}$ es la matriz de pesos de la capa de entrada, $A$ es una matriz cuyos coeficientes se determinan en el entrenamiento de la ANN y $\sigma, \varphi$ son funciones de activación tipo sigmoide.

Los pesos de la DNN se ajustan de acuerdo con las ecuaciones diferenciales matriciales dadas por $\dot{W}_{1, t}=-K_{1} P\left(\chi_{0}-\chi_{\dagger}\right) \sigma^{T}$ y $\dot{W}_{2, t}=-K_{2} P\left(\chi_{0}-\chi_{t}\right) u^{T}$, siendo $\chi_{0}$ una condición inicial, $K_{1}$ y $K_{2}$ constantes a determinar y la solución de la ecuación diferencial riccatiana, la cual se define como:

$$
A^{t} P+P A+P R P+Q=0
$$

Donde $R$ y $Q$ son matrices definidas positivas (Ortiz, 2017).

En este trabajo se emplea la DNN bajo la función sigmoide de la ecuación (1) y bajo los modelos de calibración que corresponden a regresión múltiple en series de tiempo. El objetivo es establecer un marco comparativo entre dichos procedimientos versus la DNN, y evaluar su desempeño. Este enfoque sigue los pasos señalados por (Cabrera et al., 2007) y Ortiz (2017).

En la siguiente sección, se describirá brevemente el procedimiento que se utilizará en las estimaciones de la red neuronal artificial diferencial.

\section{Metodología}

El algoritmo que se emplea en este trabajo se basa en modelos de regresión en series de tiempo para entrenar la red neuronal artificial diferencial, como se realiza en Ortiz (2017). Además, se usan dos capas de salida, donde se tienen como unidades de proceso las siguientes especificaciones:

$$
\begin{gathered}
P_{t}=\beta_{0}+\beta_{1} t+\beta_{3} P_{t-1}+u_{\mathrm{t}} \\
P_{t}=\beta_{0}+\beta_{1} V N_{t-1}+\beta_{2} A T_{t-\mathrm{m}}+\beta_{3} \mathrm{CC}_{t-\mathrm{n}}+u_{\mathrm{t}}
\end{gathered}
$$

Donde $P_{t}$ es el precio de la acción, $t$ es una variable de tendencia, $V N_{t}$ son las ventas netas, $A T_{t}$ son los activos totales, $\mathrm{CC}_{t}$ son las cuentas por cobrar y $u_{\mathrm{t}}$ es la perturbación aleatoria. Los términos $l, m, n$ representan los rezagos de ajuste según la bondad de ajuste.

El proceso de estimación se realiza en el software R-Studio y se divide en dos etapas: 
a) Las ecuaciones (4) y (5) se estiman en el período 2009-2016 mediante mínimos cuadrados generalizados (in sample) y sirven como condición inicial para las neuronas (unidades de proceso). Se elige este período muestral dada la volatilidad de la crisis financiera en el año 2008-2009.

b) Posteriormente, mediante la red neuronal, se realiza la proyección de los dos primeros trimestres de 2017 y se compara con los datos observados (out sample).

El desempeño de los modelos de regresión y de la DNN se realiza mediante el error absoluto porcentual promedio (MAPE, por sus siglas en inglés) y la raíz del error cuadrático medio (RMSE, por sus siglas en inglés), cuyas expresiones son:

$$
\begin{gathered}
R M S E=\sqrt{\frac{1}{T} \sum_{t=1}^{T}\left(y_{t}-\widehat{y_{t}}\right)^{2}} \\
M A P E=\frac{100}{T} \sum_{t=1}^{T}\left|\frac{y_{t}-\widehat{y_{t}}}{y_{t}}\right|
\end{gathered}
$$

Donde $y_{t}$ es el dato observado, $\hat{y}_{t}$ es el dato pronosticado y $\mathrm{T}$ es el total de observaciones.

En la siguiente sección, bajo el esquema anterior y con los indicadores señalados, se presentan el conjunto de estimaciones para el precio de la acción de la empresa Pfizer.

\section{Resultados preliminares}

En la primera fase de este conjunto de estimaciones, se realizó un análisis exploratorio de los precios y rendimientos de la acción Pfizer. Posteriormente, se llevaron a cabo las estimaciones con los modelos de regresión en series de tiempo y la DNN.

\section{Análisis histórico cuantitativo}

En este apartado, se revisarán las cifras de la empresa Pfizer y los conceptos de las variables más relevantes que permitan analizar la situación presente y futura de la organización. 
Los ingresos de la industria farmacéutica han ido creciendo a ritmos de $6.6 \%$ anual en promedio los últimos diez años (Pfizer, 2017). Dentro de las ventas de Pfizer, el mercado con mayor participación ha sido el estadounidense, con un promedio de $41 \%$ en los últimos cinco años, seguido por el mercado de los países desarrollados europeos con $23 \%$ y por los mercados emergentes, cuyo porcentaje ha aumentado a $21 \%$.

En relación con los costos de ventas, se han mantenido en un promedio de \$9.6 miles de millones de dólares de 2012 a 2015. El costo de ventas de 2011 a 2012 se redujo $21 \%$, pasando de $\$ 12.5$ millones de dólares a $\$ 9.8$ mil millones de dólares. Desde 2012, el costo de ventas se ha mantenido estable, presentando variaciones mínimas año con año. En 2013, se redujo $2 \%$ en comparación con 2012 debido a la pérdida de exclusividad de ciertos productos en varios mercados.

Los gastos de operación de Pfizer incluyen aquellos por concepto de administración y ventas, investigación y desarrollo, amortización, reestructura y M\&A (por sus siglas en inglés), entre otros, los cuales en conjunto representan, en promedio de los últimos cinco años, el $58 \%$ de las ventas totales. Los gastos operativos de la empresa estuvieron disminuyendo en gran medida de 2011 a 2013, incluyendo los de administración y ventas, de reestructura y M\&A.

Como puede observarse en la tabla 1, los gastos operativos pasaron de representar $56 \%$ de las ventas totales en 2014 a $62 \%$ en 2015. Esto se explica por la compra de Hospira, pues los relativos a la reestructura y M\&A se incrementaron $361 \%$. Además, los gastos de administración y ventas aumentaron $5 \%$ año con año debido a una mayor inversión en productos biofarmacéuticos y marcas del Consumer Healthcare (Damodaran, 2018).

En 2014, los gastos de operación aumentaron $5.7 \%$, en comparación con 2013 , debido a mayores gastos de investigación y desarrollo (25.7\% año con año), pese a que todos los otros gastos disminuyeron. El porcentaje que representan los gastos operativos de Pfizer de las ventas totales se ha mantenido en un rango de $50 \%$ a $62 \%$ durante los últimos cinco años, mientras que el porcentaje que representan el costo de ventas sobre las ventas se ha mantenido en un rango de $18 \%$ a $21 \%$ (Pfizer, 2017).

La inversión de Pfizer en Investigación y Desarrollo (I+D) es relevante, ya que la empresa debe innovar constantemente, pues se requieren años de trabajo para consolidar un nuevo medicamento. El departamento de I+D de Pfizer está dividido en seis grandes áreas: pequeñas y grandes moléculas, inmunología e inflamación, enfermedades cardiovasculares y metabólicas, oncología, vacunas, neurociencia y dolor y enfermedades anormales, y todas ellas permiten mayor eficiencia en el desarrollo de nuevos productos. 
Tabla 1. Cambio porcentual de gastos de operación (2011-2015; millones de dólares)

\begin{tabular}{|c|c|c|c|c|c|}
\hline Gastos de operación & 2011 & 2012 & 2013 & 2014 & 2015 \\
\hline Investigación y desarrollo & 8681 & 7482 & 6678 & 8393 & 7690 \\
\hline \% Variación a/a & $-7.6 \%$ & $-13.8 \%$ & $-10.7 \%$ & $25.7 \%$ & $-8.4 \%$ \\
\hline$\%$ de ventas netas & $14.2 \%$ & $13.7 \%$ & $12.9 \%$ & $16.7 \%$ & $15.7 \%$ \\
\hline $\begin{array}{l}\text { Gastos de ventas y } \\
\text { administración }\end{array}$ & 17581 & 15171 & 14355 & 14097 & 14809 \\
\hline \% Variación a/a & $-9.7 \%$ & $-13.7 \%$ & $-5.4 \%$ & $-1.8 \%$ & $5.1 \%$ \\
\hline$\%$ de ventas netas & $28.8 \%$ & $27.8 \%$ & $27.8 \%$ & $28.4 \%$ & $30.3 \%$ \\
\hline Gastos de amortización & 5465 & 5109 & 4599 & 4039 & 3728 \\
\hline \% Variación a/a & $1.1 \%$ & $-6.5 \%$ & $-10.0 \%$ & $-12.2 \%$ & $-7.7 \%$ \\
\hline$\%$ de ventas netas & $9.0 \%$ & $9.3 \%$ & $8.9 \%$ & $8.1 \%$ & $7.6 \%$ \\
\hline Gastos de reestructura y $M \& A$ & 2841 & 1810 & 1182 & 250 & 1152 \\
\hline \% Variación a/a & $-14.6 \%$ & $-36.3 \%$ & $-34.7 \%$ & $-78.8 \%$ & $360.8 \%$ \\
\hline$\%$ de ventas netas & $4.7 \%$ & $3.3 \%$ & $2.3 \%$ & $0.5 \%$ & $2.4 \%$ \\
\hline Otros ingresos (gastos) & 2486 & 4022 & -532 & 1009 & 2860 \\
\hline \% Variación a/a & $-42.7 \%$ & $-61.8 \%$ & $-113.2 \%$ & $-289.7 \%$ & $-183.4 \%$ \\
\hline$\%$ de ventas netas & $4.1 \%$ & $7.4 \%$ & $-1.0 \%$ & $2.0 \%$ & $5.9 \%$ \\
\hline Total de gastos operación & 37054 & 33597 & 26282 & 27788 & 30239 \\
\hline \% Variación a/a & $-11.6 \%$ & $-9.3 \%$ & $-21.8 \%$ & $-5.7 \%$ & $-8.8 \%$ \\
\hline$\%$ de ventas netas & $60.7 \%$ & $61.5 \%$ & $50.9 \%$ & $56.0 \%$ & $61.9 \%$ \\
\hline
\end{tabular}

Fuente: elaboración propia con datos de Pfizer.

Los gastos en I+D disminuyeron en 2015 debido a que se redujeron aquellos en ensayos clínicos, acuerdos de licencia, así como por el impacto favorable de $2 \%$ en divisas. En 2014, los gastos de I+D aumentaron $26 \%$ en relación con 2013 debido a un acuerdo en el que, en colaboración con Merck, se desarrollaría y comercializaría Avelumab, un tratamiento potencial contra varios tipos de cáncer. En 2013 estos gastos disminuyeron 11 \% año con año debido a que en 2012 se realizó un pago no recurrente a AztraZeneca para obtener la exclusividad y derechos del producto Nexium (Pharma, 2016).

Durante 2015, las utilidades netas disminuyeron $20 \%$ más con respecto al año anterior, a causa de una caída en sus ingresos y a un aumento en el costo de ventas y administración, principalmente por las fusiones y adquisiciones que ha realizado (Godoy, 2013). 
En relación con la estructura de capital de Pfizer, en 2015 consiste aproximadamente en $16 \%$ de deuda y $84 \%$ de capital. De hecho, según el modelo de Capital Asset Pricing Model (CAPM), el costo de capital de la empresa se ha ubicado en un rango de $5 \%$ a $8 \%$ durante los últimos seis años. En 2015, el costo de capital fue de $7.4 \%$, menor al promedio de la industria de $8.37 \%$ (Pfizer, 2017).

Adicionalmente, el WACC (Weighted Average Cost of Capital), que es el costo ponderado del capital, ha permanecido en un rango de $4.78 \%$ a $7.13 \%$ durante los últimos seis años, alcanzando un promedio de $5.9 \%$ anual (Damodaran, 2018).

Si se elabora un histograma con los precios de la acción, se infiere que el precio de esta ha estado en niveles altos con menor frecuencia, véase la gráfica 1. Los precios de las acciones de Pfizer se han valuado desde un precio mínimo de $\$ 2.75$, hasta un máximo de $\$ 37.36$, lo cual señalaba una tendencia positiva, con excepción de 2008, año de la crisis financiera.

Si se analiza el rendimiento del precio de la acción en el intervalo 20002016, se tiene una oscilación desde un $-19.4 \%$ hasta $13.8 \%$. De hecho, si se calcula el rendimiento promedio, la desviación estándar y el coeficiente de variación por períodos de cinco años, se tiene que la volatilidad de la acción se ha mostrado estable.

Gráfica 1. Histograma (en dólares)

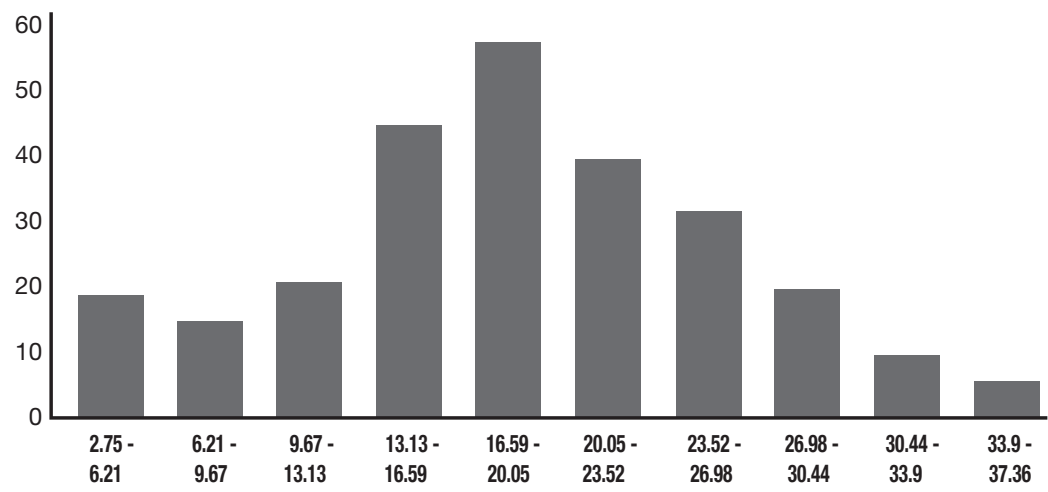

Fuente: elaboración propia con datos de Pfizer y Economática.

En la siguiente sección se presentará brevemente el marco teórico que permite vincular las variables y los indicadores que se han presentado hasta ahora. El objetivo es emplear la mayor información disponible para calibrar una red neuronal robusta y proyectar con la mayor precisión posible la evolución del precio de la acción de Pfizer en los años 2018-2020. 


\section{Datos de la muestra}

La DNN que se emplea para proyectar los rendimientos de la acción de Pfizer utiliza un esquema de propagación hacia adelante y se estima en el paquete $\mathrm{R}$.

La variable de salida es el precio trimestral (P) de cierre de la acción de Pfizer. En la gráfica 2 puede apreciarse que los movimientos anuales muestran una tendencia positiva, con excepción de 2008, año de la crisis financiera, pero el precio se recupera y sube hacia 2015. En febrero de 2015, el precio de la acción subió $10.6 \%$ anual y $10.7 \% \mathrm{~m} / \mathrm{m}$ (mes a mes), tras el anuncio de la compra de Hospira. Sin embargo, en noviembre de ese año la acción disminuyó $2.3 \%$ $\mathrm{m} / \mathrm{m}$, después de que la compañía anunció la posible fusión con Allegran. Esto indica la volatilidad del mercado y su influencia en Pfizer.

Gráfica 2. Precio de la acción de Pfizer, 1994-2017

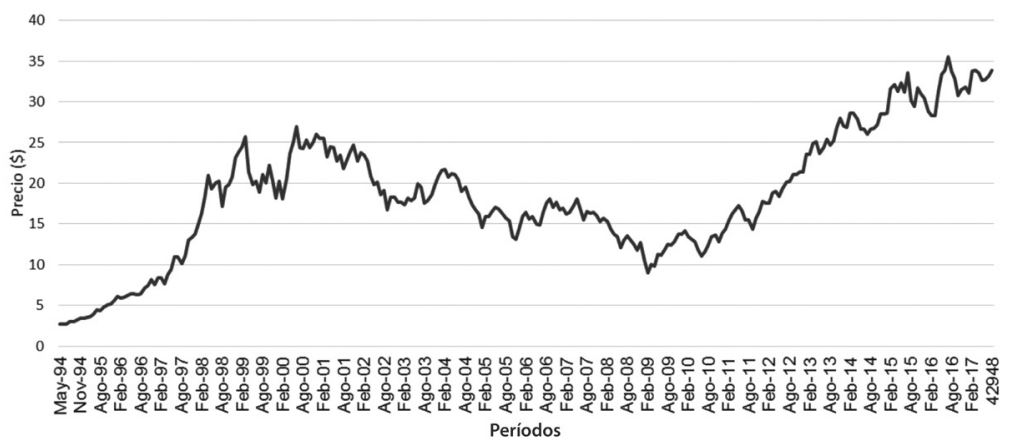

Fuente: elaboración propia.

Las variables de entrada corresponden a las series de tiempo trimestrales de ventas netas (VN), cuentas por cobrar (CC) y activos totales (AT) de la empresa Pfizer en el período 1995-2017, véase la gráfica 3.

En la gráfica 3 puede observarse que los activos promedio de la empresa han estado disminuyendo; de hecho, solo el crédito mercantil ha aumentado en este lapso. No obstante, Pfizer es una empresa que tiene activos altos en comparación con sus operaciones y utilidades. En el caso de las ventas netas, estas han mostrado una tendencia negativa, pasando de \$61035 millones de dólares en 2011 a $\$ 48851$ millones de dólares en 2015. Los años en que se reportaron los mayores decrementos en ventas fueron 2011 y 2012, debido a una disminución operativa de $\$ 4.8$ mil millones de dólares, como resultado de la pérdida de exclusividad de ciertos productos, como el Lipitor, y por el impacto desfavorable del tipo de cambio (Pfizer, 2017). 
Si se observa la gráfica de las cuentas por cobrar, se tiene una tendencia a la baja; esto es en parte debido a la disminución de los ingresos brutos de la empresa en los últimos años, así como también a un aumento de participación de mercado, pues con la adquisición de otras empresas, crece su posición en el sector (Pfizer, 2017).

Uno de los decrementos más destacados de cuentas por cobrar se da en 2013 debido a que el año anterior se efectuó la venta de una empresa de nutrición infantil a Nestlé, por lo que se traspasaron algunas de las cuentas por cobrar en la venta. En el resto de los años, las cuentas por cobrar disminuyeron aproximadamente en la misma proporción que las ventas (Pfizer, 2017).

Gráfica 3. Variables de entrada en la DNN
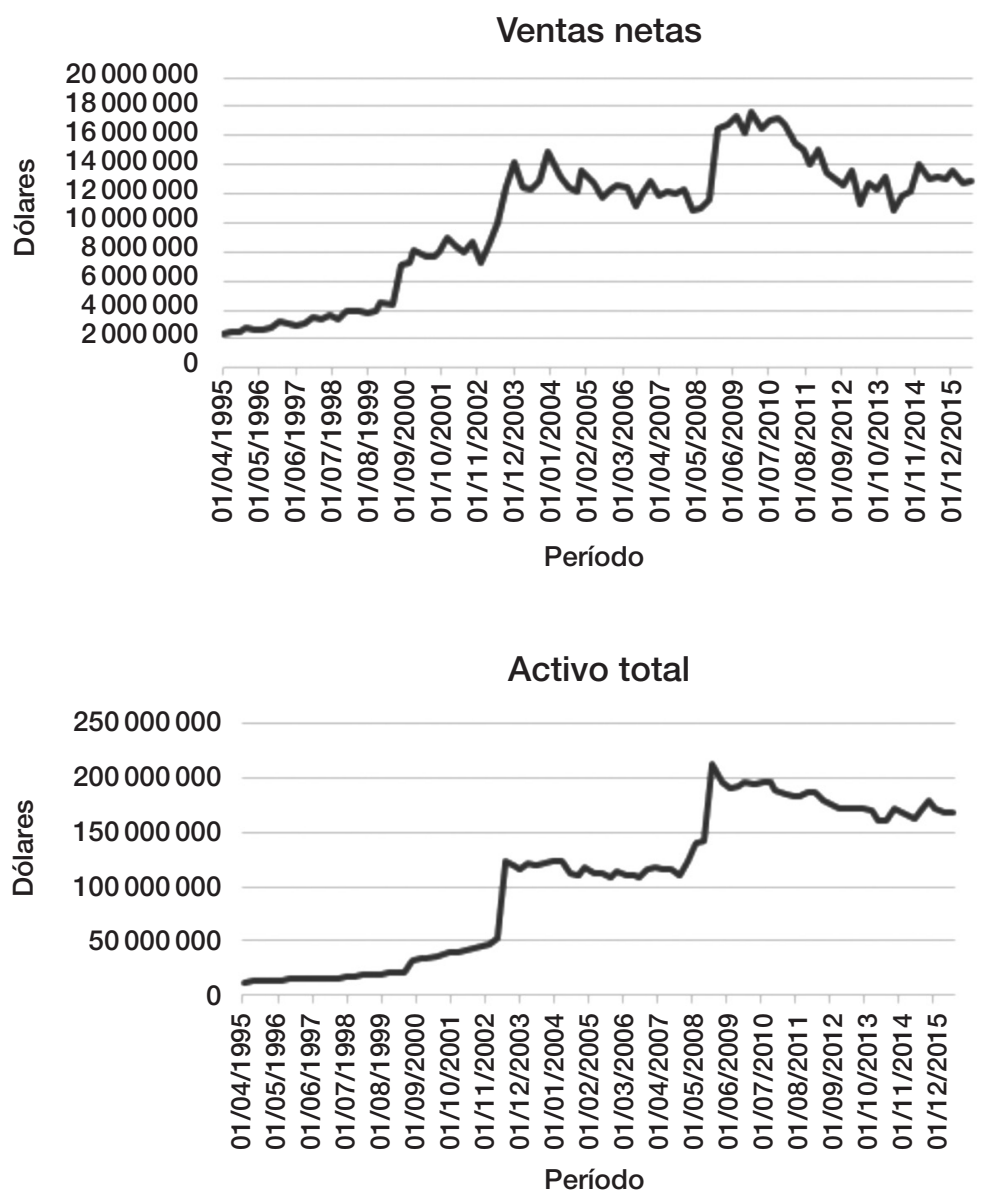


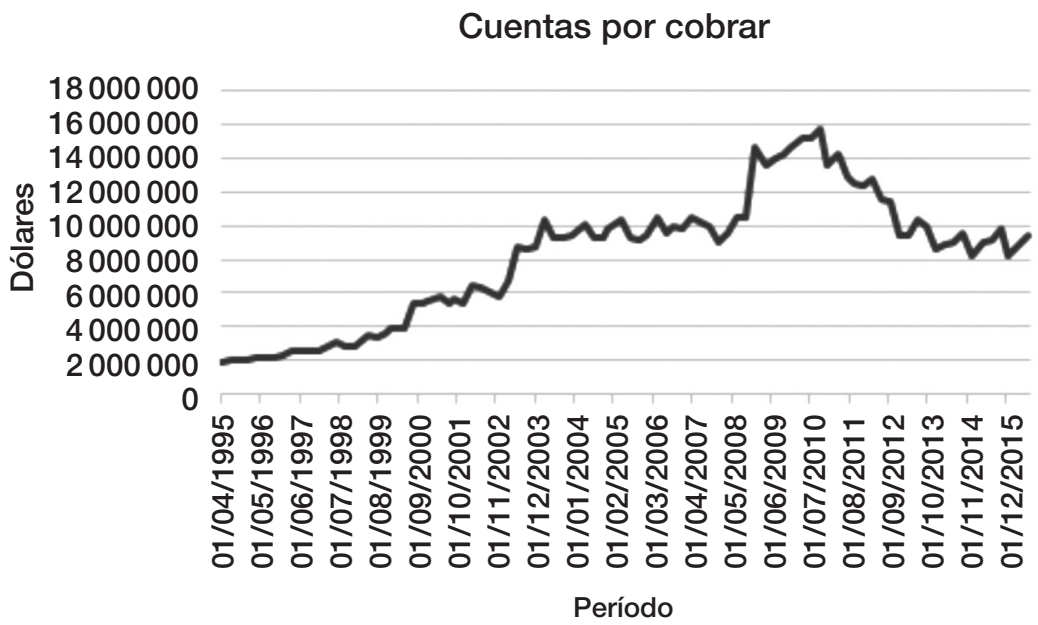

Fuente: elaboración propia.

\section{Estimación comparativa para la DNN}

A lo largo de este apartado, se muestran los cálculos necesarios para proyectar el precio de la acción de Pfizer y se presenta también una discusión sobre los resultados encontrados.

En la tabla 2 puede observarse la estimación de la ecuación (4) y cómo los coeficientes son significativos al menos al 99 \% de confianza. El componente de tendencia y el precio del trimestre anterior tienen un efecto positivo sobre el precio de la acción.

El coeficiente de determinación es ligeramente superior a $95 \%$ y, según el estadístico $\mathrm{F}$, las variables incluidas son relevantes para explicar la variable dependiente.

Es importante comentar que en el modelo de regresión uno, de la tabla 2, se tiene evidencia para afirmar que no existen problemas de autocorrelación serial ni de heterocedasticidad. Esto debido al nivel del estadístico DurbinWatson y al valor $\mathrm{p}$ de las pruebas de hipótesis. Las afirmaciones anteriores son análogas para el modelo de regresión dos de la tabla 3, salvo que el coeficiente de determinación es $84.18 \%$.

Las ecuaciones estimadas de las tablas 2 y 3 se emplearán como punto de partida dentro de la DNN. De esta forma, se iniciará con el proceso de realimentación para proyectar el precio de la acción de la empresa Pfizer. 
Tabla 2. Modelo de regresión uno

\begin{tabular}{|l|r|r|r|r|}
\hline Variable & Coeficiente & $\begin{array}{c}\text { Error } \\
\text { estándar }\end{array}$ & Estadístico t & \multicolumn{1}{|c|}{ Valor p } \\
\hline C & -18.0403 & 5.4488 & -3.3109 & 0.0025 \\
\hline @TREND & 0.4057 & 0.1162 & 3.4903 & 0.0016 \\
\hline PRECIO(-1) & 0.4902 & 0.1514 & 3.2389 & 0.0030 \\
\hline R-cuadrado & 0.9527 & & C. I. Akaike & 3.9579 \\
\hline R-cuadrado ajustado & 0.9494 & & C. I. Schwarz & 4.0953 \\
\hline Estadístico F & 291.8431 & & C. I. HQ & 4.0034 \\
\hline Valor p & 0.0000 & & Durbin Watson & 2.0063 \\
\hline Test de heteroscedasticidad & & & Test Ljung-Box & \\
\hline Estadístico F & 0.2474 & & Estadístico JB & 6.2575 \\
\hline Valor p & 0.6226 & & Valor p & 0.2823 \\
\hline
\end{tabular}

Fuente: elaboración propia.

Tabla 3. Modelo de regresión dos

\begin{tabular}{|l|r|r|r|r|}
\hline Variable & Coeficiente & $\begin{array}{c}\text { Error } \\
\text { estándar }\end{array}$ & Estadístico t & Valor p \\
\hline VN(-3) & 0.000000835 & 0.000000372 & 2.2432 & 0.0324 \\
\hline AT(-1) & 0.000000294 & 0.000000038 & 7.8623 & 0.0000 \\
\hline CC(-1) & -0.000004530 & 0.000000392 & -11.5692 & 0.0000 \\
\hline C & 10.201670000 & 5.256472000 & 1.9408 & 0.0617 \\
\hline R-cuadrado & 0.8418 & & C. I. Akaike & 5.2873 \\
\hline R-cuadrado ajustado & 0.8260 & & C. I. Schwarz & 5.4668 \\
\hline Estadístico F & 53.2213 & & C. I. HQ & 5.3485 \\
\hline Valor p & 0.0000 & & Durbin Watson & 2.0020 \\
\hline Test de heteroscedasticidad & & & Test Ljung-Box & \\
\hline Estadístico F & 0.0239 & & Estadístico JB & 4.2388 \\
\hline Valor p & 0.8782 & & Valor p & 0.3751 \\
\hline
\end{tabular}

Fuente: elaboración propia.

Al comparar el desempeño de los modelos de regresión, se encuentra que el modelo uno tiene un error más pequeño de pronóstico, de $6.77 \%$ dentro de la muestra contra $14.65 \%$ del modelo dos. El resultado es similar fuera de la muestra, donde el modelo uno tiene un error de $6.88 \%$ 
versus $14.98 \%$ de la otra ecuación. La conclusión es la misma si se emplea como criterio el error cuadrático medio.

Sin embargo, cuando se conjugan estos modelos dentro del esquema de las unidades de proceso de la DNN, se tiene un error de pronóstico fuera de la muestra de $4.53 \%$, más pequeño que ambas ecuaciones.

En la tabla 5 se presentan las proyecciones para el precio de la acción de Pfizer desde el tercer trimestre de 2017 hasta el último trimestre de 2020. Con base en esos cálculos, se tienen el rendimiento proyectado de la acción de la empresa y el intervalo de confianza para el precio (99\% de confianza).

Puede apreciarse que el rendimiento va disminuyendo conforme transcurren los trimestres, a pesar de la tendencia al alza. Además, el precio mínimo que se estima para la acción es \$41.06, con un precio máximo de \$53.04 hacia 2020 .

Tabla 4. Bondad de ajuste

\begin{tabular}{|l|c|c|c|c|c|c|}
\hline & Modelo 1 & \multicolumn{2}{c|}{ Modelo 2 } & \multicolumn{2}{c|}{ Modelo 3 } \\
\hline & $\begin{array}{c}\text { In } \\
\text { sample }\end{array}$ & $\begin{array}{c}\text { Out } \\
\text { sample }\end{array}$ & $\begin{array}{c}\text { In } \\
\text { sample }\end{array}$ & $\begin{array}{c}\text { Out } \\
\text { sample }\end{array}$ & $\begin{array}{c}\text { In } \\
\text { sample }\end{array}$ & $\begin{array}{c}\text { Out } \\
\text { sample }\end{array}$ \\
\hline RMSE & 1.5941 & 2.3541 & 3.0972 & 4.4251 & NA & 1.4751 \\
\hline MAPE & $6.77 \%$ & $6.88 \%$ & $14.65 \%$ & $14.98 \%$ & NA & $4.53 \%$ \\
\hline
\end{tabular}

Fuente: elaboración propia.

Los resultados de la tabla 5 se muestran mediante una gráfica de abanico (véase gráfica 4), donde se aprecia cómo el intervalo de confianza es asimétrico, es decir, está sesgado hacia precios altos. También se aprecia cómo van extendiéndose los intervalos de confianza al transcurrir los trimestres hacia el año 2020.

Tabla 5. Proyecciones del precio de la acción de Pfizer hacia 2020

\begin{tabular}{|c|c|c|c|}
\hline Período & $\begin{array}{c}\text { Precio } \\
\text { proyectado (\$) }\end{array}$ & $\begin{array}{c}\text { Rendimiento } \\
\text { estimado }\end{array}$ & $\begin{array}{c}\text { Intervalo de } \\
\text { confianza (99\%) }\end{array}$ \\
\hline $2017 Q 3$ & 34.08 & $6.79 \%$ & $(30.58,39.08)$ \\
\hline $2017 Q 4$ & 35.58 & $4.31 \%$ & $(32.16,40.45)$ \\
\hline $2018 Q 1$ & 36.72 & $3.16 \%$ & $(31.86,42.11)$ \\
\hline $2018 Q 2$ & 37.69 & $2.59 \%$ & $(33.19,43.26)$ \\
\hline $2018 Q 3$ & 38.57 & $2.31 \%$ & $(33.17,44.23)$ \\
\hline $2018 Q 4$ & 39.40 & $2.15 \%$ & $(35.14,44.61)$ \\
\hline $2019 Q 1$ & 40.22 & $2.05 \%$ & $(34.05,45.71)$ \\
\hline
\end{tabular}




\begin{tabular}{|c|c|c|c|}
\hline Período & $\begin{array}{c}\text { Precio } \\
\text { proyectado (\$) }\end{array}$ & $\begin{array}{c}\text { Rendimiento } \\
\text { estimado }\end{array}$ & $\begin{array}{c}\text { Intervalo de } \\
\text { confianza (99 \%) }\end{array}$ \\
\hline $2019 Q 2$ & 41.03 & $1.98 \%$ & $(37.09,46.83)$ \\
\hline $2019 Q 3$ & 41.83 & $1.93 \%$ & $(37.95,46.83)$ \\
\hline $2019 Q 4$ & 42.62 & $1.89 \%$ & $(38.24,48.62)$ \\
\hline $2020 Q 1$ & 43.42 & $1.85 \%$ & $(38.65,50.12)$ \\
\hline $2020 Q 2$ & 44.22 & $1.82 \%$ & $(38.97,50.19)$ \\
\hline $2020 Q 3$ & 45.01 & $1.78 \%$ & $(39.71,52.19)$ \\
\hline $2020 Q 4$ & 45.81 & $1.75 \%$ & $(41.06,53.04)$ \\
\hline
\end{tabular}

Fuente: elaboración propia.

Gráfica 4. Proyecciones del precio de la acción 2017-2020

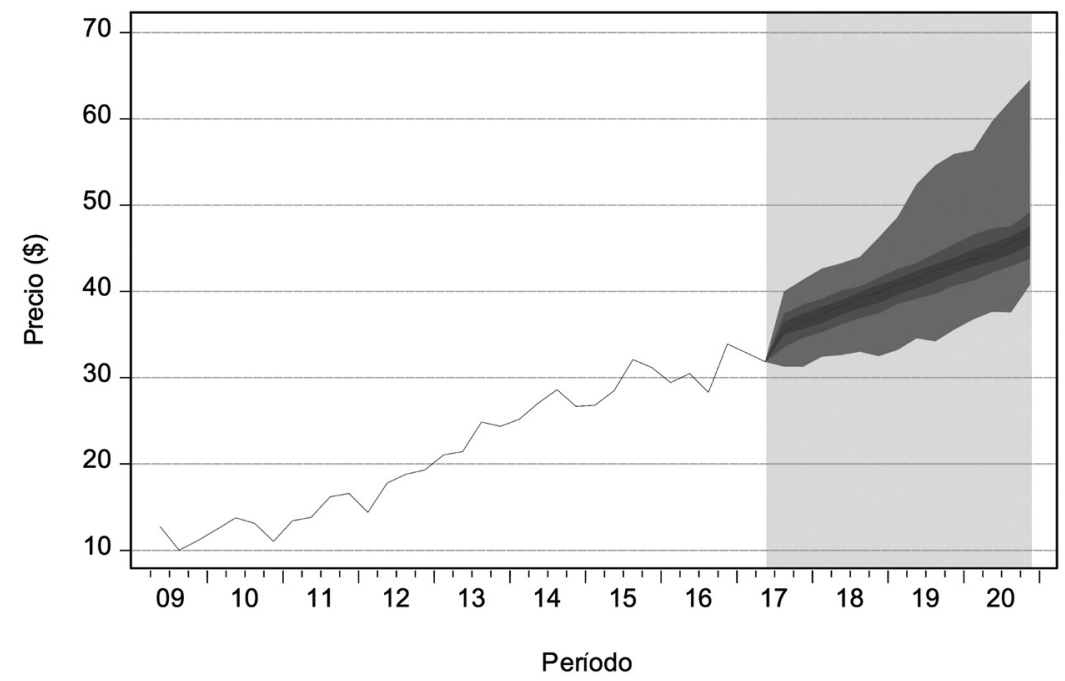

Fuente: elaboración propia.

\section{Conclusiones}

En este trabajo, se realizó un análisis cualitativo de la empresa Pfizer y se eligieron las ventas netas (VN), activos totales (AT) y cuentas por cobrar (CC) como variables relevantes para el precio de la acción. Estas variables se conjuntaron en un modelo de regresión en series de tiempo, que sirvió de comparación con un modelo en tendencia del precio de la acción. Ambas 
especificaciones se emplearon como insumos para la implementación de una red neuronal artificial diferencial. De esta manera, se realizó la proyección del precio en el período 2016-2020 y se pudieron establecer comparaciones entre la red neuronal y los métodos convencionales.

Concretamente, las estimaciones y resultados que se encontraron señalan las ventajas de las redes neuronales, en cuanto al pronóstico, pues bajo el enfoque de aprendizaje adaptativo de la red neuronal el error de pronóstico out sample para el precio de la acción de Pfizer es $4.53 \%$, un nivel más bajo que el error estimado bajo los modelos de regresión usuales.

Esto sin duda es de gran utilidad para la empresa Pfizer, ya que la proyección del precio de la acción podría monitorearse y calibrarse constantemente, y considerar escenarios con horizontes de corto, mediano y largo plazo para la toma de decisiones.

Por otro lado, debe comentarse que las principales limitaciones de este trabajo son la disponibilidad de información con frecuencia menor a un trimestre y la naturaleza heurística de la red neuronal. En el primer caso, si se tuvieran variables financieras con frecuencia diaria, podría ampliarse la especificación de la red neuronal para proyecciones diarias del precio de la acción. En el segundo caso, como el proceso es heurístico, no pueden establecerse relaciones concretas entre las variables, solamente es posible procesarlas empíricamente para generar pronósticos con bajo error in sample y out sample.

Por ello, las líneas de investigación futuras se propondrían analizar las proyecciones de la red neuronal con datos de periodicidad más corta, en la medida de lo posible, para otras empresas o activos financieros. Asimismo, otra área de oportunidad es el análisis de las proyecciones bajo diferentes insumos de entrada en la red neuronal, ya que podría mejorarse la precisión de los pronósticos aun más y contribuir de esa manera a la toma de decisiones. 


\section{Los autores}

Alfonso Aja Kindelan es licenciado en Economía y licenciado en Finanzas y Contaduría Pública por la Universidad Anáhuac México. Ha realizado estancias de estudios e investigación en Estados Unidos. Socio fundador de El Pescau y El Mar Abierto, empresas de reciente creación y ascenso rápido en la distribución y comercialización de pescados y diversos productos marinos.

Correo electrónico: aja_45@hotmail.com

Leovardo Mata Mata es doctor en Ciencias Financieras por EGADE Business School, Tecnológico de Monterrey. Maestro en Economía por El Colegio de México. Licenciatura en Física y Matemáticas en la Escuela Superior de Física y Matemáticas del Instituto Politécnico Nacional. Ha impartido seminarios y diplomados en diversas instituciones, entre las que sobresalen la Universidad Anáhuac, El Colegio de México, el Tecnológico de Monterrey, el Instituto Politécnico Nacional, la Universidad Iberoamericana y la Universidad Autónoma del Estado de México. Actualmente es profesor investigador de la Facultad de Economía y Negocios de la Universidad Anáhuac México, consultor asociado en V\&M Servicios de Consultoría S.C. y miembro del Sistema Nacional de Investigadores.

Correo electrónico: leovardo.mata@anahuac.mx

Jaime Humberto Beltrán Godoy es doctor en Ciencias Administrativas por EGADE Business School, Tecnológico de Monterrey. Maestro en Ingeniería con Especialidad en Calidad y Productividad y Licenciatura en Ingeniería Industrial por el Tecnológico de Monterrey. De 2012 a 2015 fue subdirector de área en la Dirección General de Informática de la Comisión Nacional Bancaria y de Valores. Ha impartido seminarios y diplomados en diversas instituciones entre las que sobresalen el Tecnológico de Monterrey, el Instituto Tecnológico de Ciudad Madero, la Universidad del Noreste y el Instituto Tecnológico Superior de Pánuco. Actualmente, es profesor investigador de la Facultad de Economía y Negocios de la Universidad Anáhuac México y miembro del Sistema Nacional de Investigadores.

Correo electrónico: jaime.beltrang@anahuac.mx 


\section{Referencias bibliográficas}

Chen, L. (21 de diciembre de 2015). "The Most Profitable Industries in 2016 Industry». Forbes. Recuperado el 1 de febrero de 2017 de: http:// www.forbes.com/sites/liyanchen/2015/12/21/the-most-profitableindustries-in-2016/\#686700cd7a8b

Damodaran, A. (2018). Data: Current, Damodaran online (en línea). Recuperado el 26 de enero de 2018 de: http://pages.stern.nyu.edu/ adamodar/

Evaluate Pharma Ltd. (2016). World Preview 2015, Outlook to 2020 (en línea). Recuperado el 8 de septiembre de: http://info.evaluategroup.com/ rs/607-YGS-364/images/wp15.pdf

Foix, C. y Weber, R. (2007). «Pronóstico del precio del cobre mediante redes neuronales». Ingeniería de Sistemas, 21 (1): 63-75.

Godoy, E. (9 de noviembre de 2013). «La guerra de los medicamentos». Proceso. Recuperado el 1 de marzo de 2017 de: http://www.proceso.com. $\mathrm{mx} / 357431 /$ la-guerra-de-los-medicamentos-2

Hornik, K., Stinchcombe, M. y White, H. (1989). «Multilayer Feedforward Networks and Universal Approximations». Neural Networks 2 (1): 359366.

Landassuri, V., Bustillo, C., Carbajal, J. y Sánchez, L. P. (20-23 de noviembre de 2013). «Single-step-ahead and Multi-step-ahead Prediction with Evolutionary Artificial Neural Networks». En Ruiz-Shulcloper, J., Sanniti di Baja, G. Progress in Pattern Recognition, Image Analysis, Computer Vision and Applications. 18th Iberoamerican Congress, CIARP 2013, Proceedings, Part II. La Habana, Cuba.

Lara, A. (2009). Medición y control de riesgos financieros. México, Limusa.

León, L. M., Landassuri, V. M., Orozco, H. y Quintana, M. (2018). «Predicción del IPC mexicano combinando modelos econométricos e inteligencia artificial». Revista Mexicana de Economía y Finanzas, 13 (4): 603-629.

Londoño, C., Lopera, M. y Restrepo, S. (2010). «Teoría de precios de arbitraje. Evidencia empírica para Colombia a través de redes neuronales». Revista de Economía del Rosario, 13 (1): 41-73.

Moghaddam, A. H., Moghaddam, M. H. y Esfandyari, M. (2016). «Stock market index prediction using artificial neural network». Journal of Economics, Finance and Administrative Science, 21 (41): 89-93.

Misas, M., López, E. y Borrero, P. (2002). «La inflación en Colombia: una aproximación desde redes neuronales». Ensayos sobre política económica, 41 (42): pp. 143-214.

Pao, H. T. (2008). «A comparison of neural network and multiple regression analysis in modeling capital structure». Expert Systems with Applications, 35 (3): 720-727.

Ortiz, F. (2017). «Pronóstico de precios de petróleo: una comparación entre modelos GARCH y redes neuronales diferenciales». Investigación económica, 76 (300): 105-126. 
Ortiz, F. O., Llanos, A. I. C. y Herrera, F. L. (2013). «Pronóstico de los índices accionarios DAX y S\&P 500 con redes neuronales diferenciales». Contaduría y Administración, 58 (3): 203-225.

Ospina, R. M. y Zamprogno, B. (2003). «Comparación de algunas técnicas de pronóstico y análisis de series de tiempo». Revista Colombiana de Estadística, 26 (2): 129-157.

Pfizer (2017). ¿Quiénes somos? (sitio de internet). Recuperado de: https:// www.pfizer.com.mx/content/historia-de-pfizer\#.V83DvZjhCUk

«Pfizer compra Allergan y crea la mayor farmacéutica 2020» (23 de noviembre de 2015). Forbes. Recuperado el 20 de enero de 2018 de: https:// www.forbes.com.mx/pfizer-y-allergan-crean-la-mayor-farmaceuticadel-mundo/

Pharma (2016). 2015 Profile: Biopharmaceutical Research Industry (en línea). Recuperado el 1 de febrero de: http://www.phrma.org/sites/default/ files/pdf/2015_phrma_profile.pdf

Ross, S., Westerfield, R. y Jaffe, J. (2012). Finanzas corporativas. México: McGraw Hill.

Salazar, M. A. y Cabrera, M. (2007). Pronóstico de demanda por medio de redes neuronales artificiales. Ingenierías, 10 (35): 6-12.

Global Pharmaceutical Industry - Statistics \& Facts (2017). The Statistics Portal (base de datos en línea). Recuperado el 29 de enero de 2018 de: http://www.statista.com/topics/1764/global-pharmaceutical-industry/

Toro, O., Mejía, G. y Salazar, I. (2004). «Pronóstico de ventas usando redes neuronales». Scientia et technica, 10 (26): 1-19.

Tricker, B. (2009). Corporate Governance: Principles, Policies, and Practices. Nueva York: Oxford University Press.

Sirghi, N. (2012). «Perspectives in the theory of the firm». Economic Studies, 2 (1): 134-142.

Venegas, F. (2013). Riesgos financieros y económicos. Productos derivados y decisiones económicas bajo incertidumbre. México: Cengage Learning.

Villada, F., Muñoz, N. y García, E. (2012). «Aplicación de las redes neuronales al pronóstico de precios en el mercado de valores». Información tecnológica, 23 (4): 11-20.

White, H. (1988). «Economic prediction using Neural Networks: The case of IBM Daily Stock returns». Proceedings of the IEEE International Conference on Neural Networks, San Diego, vol. II: 451-459. 